\title{
Preliminary Study on High-sensitive Diffraction Enhanced Imaging at BSRF
}

\author{
Qingxi Yuan ${ }^{1, *}$, Jin Zhang ${ }^{1,2}$, Yu Chen ${ }^{1}$, Kai Zhang ${ }^{1}$, Wanxia Huang ${ }^{1}$, Peiping Zhu ${ }^{1}$ \\ 1. Institute of High Energy Physics, Chinese Academy of Sciences, Beijing, P. R. China. \\ 2. University of Chinese Academy of Sciences, Beijing, P. R. China. \\ * Corresponding author, email_yuanqx@ihep.ac.cn
}

As one of the main phase contrast imaging methods, diffraction enhanced imaging (DEI) has attracted interest in many scientific fields especially in biomedical applications [1-3] since it was proposed by Chapman et al in 1997 [4] . But for live biological samples or samples with small density variation, pre-processing such as supplement of contrast enhancement agent or dipping sample in formalin solution has to be used to get high imaging contrast, resulting in the limitation of in-situ study about those samples. For this issue, high-sensitive imaging method might be a possible solution. On the other hand, rocking curve is the main feature of DEI setup and its width comes from the type of crystal diffraction plane used. Although DEI imaging sensitivity depends on several factors, high-imaging-contrast can be expected using crystals with smaller width of rocking curve [5]. Based on these points, an attempt for high-sensitive DEI has been carried out based on selection of different high-index diffraction plane of silicon crystal.

With the rocking curve going narrower, the usable photon flux will go lower and the needed stability of the setup will go higher. Under the consideration of relevant factors, diffraction plane of Si (400) and Si (333) was selected for the study of actually acquired image contrast with respect to the width of rocking curve and the stability of imaging setup. For high-sensitive DEI setup, there are two key points, one is the rotational accuracy of pitch angle for analyzer crystal, and the other one is rocking curve width acquired during the experiment should be close to theoretical value. Finally, KTG-15D (Kohzu) rotation stage with 0.005 arcsec resolution was selected for analyzer crystal pitch, low-stress technique was used for crystal processing and special design was used to minimize clamping stress of crystal.

DEI experiments were carried out at beam line 4W1A of Beijing Synchrotron Radiation Facility (BSRF) [6]. The selected samples in the experiments are standard sample and biological samples. Standard sample is a nylon fiber with diameter of $0.54 \mathrm{~mm}$. Biological samples are mouse liver treated by formalin and dry beetle sample. Multiple-image radiography (MIR) method [7] and cosine fitting radiography (CFR) method [8] were used to extract absorption information, refraction information and scattering information of each sample. For all samples, MIR and CFR data were acquired at $15 \mathrm{keV}$ with $\mathrm{Si}(111)$, $\mathrm{Si}(333)$ and $\mathrm{Si}(400)$ respectively. 721 projections were collected within $180^{\circ}$ angle range for CT reconstruction with analyzer crystal tuned at the peak of rocking curve.

Based on the gray value of certain pixels of MIR background image, the actual rocking curve was obtained at $15 \mathrm{keV}$ using Gaussian fitting. The FWHM value of experimental rocking curve (figure 1) was 5.99", 2.28" and 1.11" respectively for three types of silicon crystals, which is very close to theoretical value 5.20", 2.36" and 1.14" calculated by XOP software. Figure 1 also gave the edge visibility of standard sample at different rocking curve position calculated using gray value of pixels from a certain area of projected images, maximum edge visibility resulting in $0.0683,0.1186$ and 0.2170 
respectively.
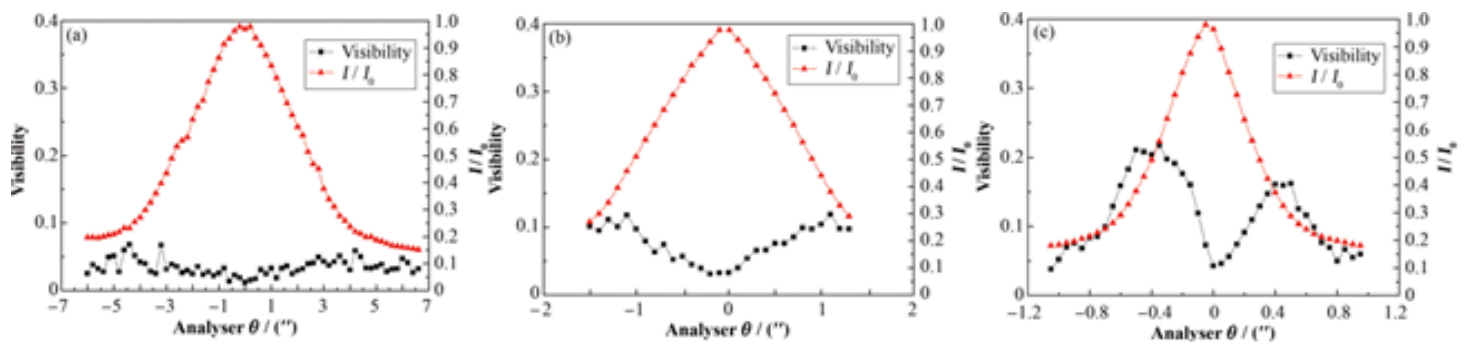

Figure.1. The experimental rocking curve of DEI setup with $\mathrm{Si}$ (111) (a), Si (400) (b), Si (333) (c) and corresponding edge visibility of standard sample.

Figure 2 shows three peak images of mouse liver obtained from three different DEI setup respectively. From the indicated area, more details can be acquired with the increasing of crystal index.

The reconstructed slices of beetle sample also give the same results as that from figure 2 .

Based on the preliminary imaging results of standard sample and

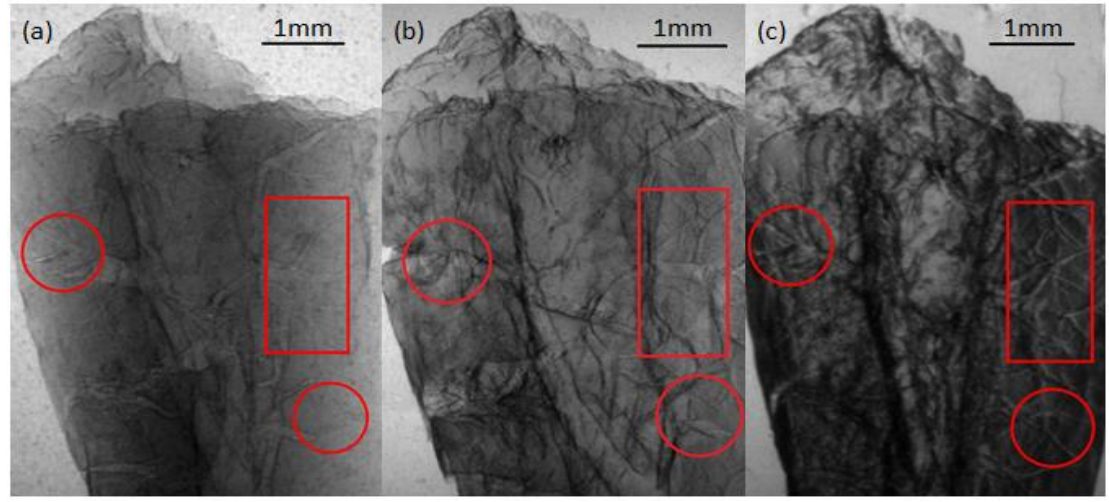

Figure.2. Peak images of sample mouse liver obtained using DEI set up with $\operatorname{Si}(111)$ (a), $\operatorname{Si}(400)$ (b) and $\operatorname{Si}(333)$ (c) respectively.

biological samples, high-sensitive imaging results can be obtained with high-index silicon crystal, showing the possibility of getting high imaging contrast for biological samples in real status and samples with small density variation. Further study is also needed to get contrast resolving ability for different crystals and to ascertain favorable setup for different types of sample.

Reference:

[1] Jun Li et al, J. Anat. 202(2003) p463

[2] S Fiedler1 et al, Phys. Med. Biol. 49 (2004) p175

[3] Yunzhe Zhao et al, PNAS 45(2012) p18290

[4] Chapman D et al, Phys. Med. Biol. 42(1997) p2015

[5] Luigi Rigon et al, Proceedings of SPIE 4682 (2002) p255

[6] Qingxi Yuan et al, Radiation Physics and Chemistry 75(2006) p1990

[7] Miles N Wernick et al, Phys. Med. Biol. 48 (2003) p3875

[8] Li Pan-Yun et al, Chin. Phys. B 24 (2015) 068704 